\title{
Psychedelic View of Fluid in Porous Materials
}

\author{
Video from a new technique reveals the chaotic motion of a fluid flowing \\ through a pile of gravel.
}

By David Ehrenstein

W

hen polluted water flows through an aquifer, researchers would like to know how the pollutant concentration changes and becomes diluted over time. A new method reveals the dilution process of a substance flowing through a porous material like rock, where direct imaging is difficult [1]. The technique involves sending a dye-laced fluid into the material and then reversing the flow while observing the chaotic spreading of the emerging dye. The researchers who developed the method say it could be used to study many situations, such as contaminated water flowing through soil or reagents flowing through columns of beads in industrial reactors.
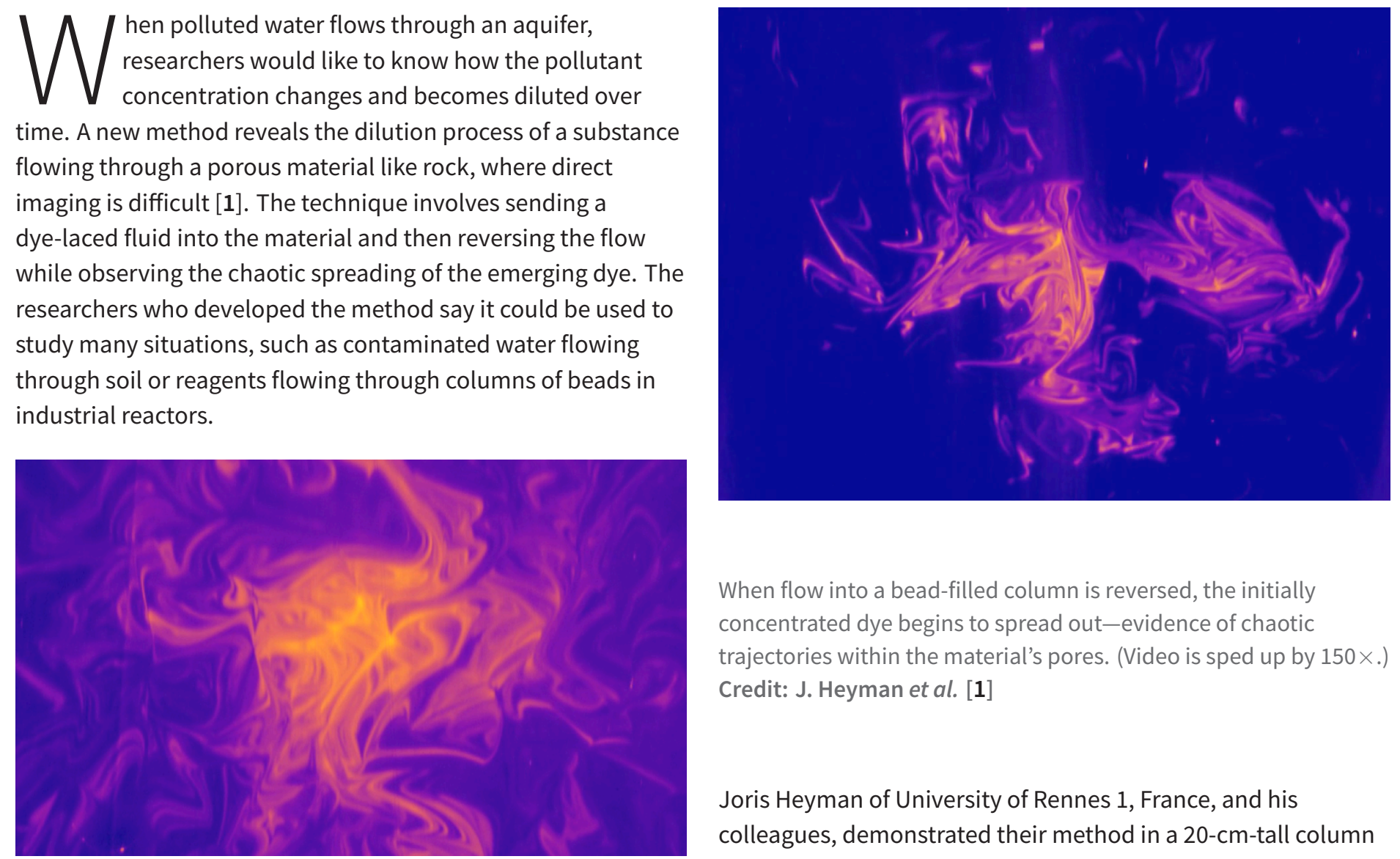

When flow into a bead-filled column is reversed, the initially concentrated dye begins to spread out-evidence of chaotic trajectories within the material's pores. (Video is sped up by $150 \times$.) Credit: J. Heyman et al. [1]

Flow echo. The dye in a fluid spreads out as it flows into-and later flows back out of-a porous material. The experiment could provide new information on the dilution of contaminants in pollution spills. (See video below.)

Credit: J. Heyman et al. [1]

Joris Heyman of University of Rennes 1, France, and his colleagues, demonstrated their method in a $20-\mathrm{cm}$-tall column of either millimeter-wide beads or gravel. First, a viscous mixture of water and glycerin flowed slowly and continuously downward through the pores in the column. Then the team injected a dye into the flow from a needle above the porous material. After the dye had penetrated all the way through the column, the team shut off the needle and reversed the flow direction. The emerging dye was illuminated by a "laser sheet" just above the top of the material, allowing a video camera to capture the horizontal spreading of the dye. 
When the flow was reversed, the first bit of dye to emerge-corresponding to the earliest frames of the video-was highly concentrated in the center. Later frames recorded increasingly chaotic patterns within fluid that returned from deeper in the column. The researchers showed-using theory, simulations, and experiments-that the video provides detailed information about the dilution process of the dye within the porous material. Even though the flow was slow and not turbulent, the pores in the column generated chaotic trajectories for the dye that facilitated dilution.

David Ehrenstein is the Focus Editor for Physics.

\section{REFERENCES}

1. J. Heyman et al., "Scalar signatures of chaotic mixing in porous media," Phys. Rev. Lett. 126, 034505 (2021). 